\section{A solution to the problem of ion confounding in experimental biology}

To the editor: Understanding ion-specific effects is a central theme of biology. But because ions are generally manipulated through the use of salts, the complication of 'ion confounding' is introduced. Ion confounding occurs when the ion(s) of interest are covaried with the covaried ion associated with the salts used; that is, changing the concentration of a single cation or anion using a single salt results in a simultaneous change in the associated co-ion. If the concentration of an ion is not varied independently, then the main effect associated with that ion is indistinguishable from or confounded with, the effect(s) caused by changing the concentrations of the other ions. Identifying ion confounding requires converting reported salt formulations, including ions introduced via $\mathrm{pH}$ adjustments, to corresponding ion concentrations. If there are deviations of ion type and/or concentration other than those specified by the experimental design, then ion confounding has occurred. Ion confounding is extremely common in disciplines that study ion-specific effects, but is particularly apparent in plant nutrition studies, as the complex mineral-ion mixtures used are the primary nutritive source of these organisms (Table 1$)^{1}$.

To avoid ion confounding, it is necessary to be able to calculate a salt mix, or 'recipe', that achieves any given ionic formulation. This can be easily solved as a linear programming problem that optimizes the allocation of mixture components (the ions) from a set of salts. The linear function, presented as a minimization, can 20 be used to determine the types and concentrations of salts, acids and bases to achieve a particular ionic solution:

minimize

$$
c_{1} X_{1}+c_{2} X_{2}+\ldots+c_{n} X_{n}
$$

where $c$ is a known weighting coefficient, and $X$ is the unknown amount of an individual salt,

subject to $a_{i 1} X_{1}+a_{i 2} X_{2}+\ldots+a_{i n} X_{n}\{\leq,=, \geq\} b_{i}(i=1,2, \ldots m)$

$$
X_{1}, \ldots X_{n} \geq 0
$$

where $a$ are known coefficients of ion proportionality for each salt $X$ that contributes an ion defined by the target ion concentration $b$; a non-negative constraint ensures positive values. Equation (1) results
Table 1 | Ion confounding of the hydroponic nutrient solutions

\begin{tabular}{llllllll}
\hline Solution & $\mathrm{pH}$ & $\mathrm{NO}_{3}{ }^{-}$ & $\mathrm{PO}_{4}^{3-}$ & $\mathrm{K}^{+}$ & $\mathrm{Ca}^{2+}$ & $\mathrm{Mg}^{2+}$ & $\mathrm{SO}_{4}{ }^{2-}$ \\
\hline Control & 4.44 & 15 & 1 & 6 & 5 & 2 & 2.05 \\
Lacking $\mathrm{N}$ & 4.51 & $0^{\mathrm{a}}$ & 1 & 5 & 2.5 & 2 & 6.55 \\
Lacking $\mathrm{PO}_{4}{ }^{3-}$ & 4.54 & 14 & $0^{\mathrm{a}}$ & 6 & 4 & 2 & 2.05 \\
Lacking $\mathrm{K}^{+}$ & 4.43 & 10 & 1 & $0^{\mathrm{a}}$ & $\mathbf{5 . 5}$ & 2 & 2.05 \\
Lacking $\mathrm{Ca}^{2+}$ & 4.45 & 5 & 1 & 6 & $0^{\mathrm{a}}$ & 2 & 2.05 \\
Lacking $\mathrm{Mg}^{2+}$ & 4.44 & 14 & 1 & 10 & 4 & $0^{\mathrm{a}}$ & 1.55 \\
Lacking $\mathrm{SO}_{4}{ }^{2-}$ & 4.27 & 18 & 1 & $\mathbf{7}$ & 4 & 2 & $0^{\mathrm{a}}$ \\
\hline
\end{tabular}

Ion confounding is illustrated by calculating the ion concentrations of the popular salt formulations developed by Hoagland and Arnon ${ }^{1}$ for determining ion-specific effects on hydroponically grown plants. When the formulations are viewed in this manner the confounding becomes clear. For example, the formulation 'lacking $\mathrm{Mg}^{2+1}$ does not just change the $\left[\mathrm{Mg}^{2+}\right]$ as would be required to determine the effect(s) of this ion, but compared to the control solution ion levels also changes the $\left[\mathrm{NO}_{3}{ }^{-}\right],\left[\mathrm{K}^{+}\right],\left[\mathrm{Ca}^{2+}\right]$ and $\left[\mathrm{SO}_{4}{ }^{2-}\right]$. Consequently, the measured responses cannot be attributed to $\mathrm{Mg}^{2+}$ alone, but will be a product of the combined changes of multiple ions; the $\mathrm{Mg}^{2+}$ main effect is now confounded with the effect(s) of the covaried ions. Only major mineral ions are shown; $\mathrm{Cl}^{-}, \mathrm{Fe}^{2+}, \mathrm{Zn}^{2+}$ and $\mathrm{Cu}^{2+}$ are also confounded. $\mathrm{pH}$ calculated using MINEQL+ ver 4.5 (Environmental Research Software, Hallowell, Maine, USA) chemical equilibrium modeling system. Ion levels are in mM. Confounded ions are in bold.

a Ions being varied.

in a list of the type and amount of salts to achieve the exact ion concentrations specified by $b$ (Supplementary Methods online). A software application for making these calculations and generating associated pipetting tables is available from the authors.

Equation (1) provides the means to generate exact solutions of complex ion mixtures. Thus, ions can and should be manipulated as independent factors in statistically valid experiments (Supplementary Note online). The overarching impact of ion confounding is that our understanding of ion-specific effects on biological processes is primarily based on flawed experimental designs. This implies that the most basic ion-specific effects on biological responses have never been properly characterized (Supplementary Discussion online). The approach presented here provides the means to overcome this fundamental hindrance to valid experimental study of ion-specific effects.

Note: Supplementary information is available on the Nature Methods website.

\section{Randall P Niedz \& Terence J Evens}

Agricultural Research Service, US Horticultural Research Laboratory, Fort Pierce, Florida 34945, USA.

e-mail: rniedz@ushrl.ars.usda.gov

1. Hoagland, D.R. \& Arnon, D.I. The water-culture method for growing plants without soil. Univ. Calif. Coll. Agric. Exp. Stn. Circ. 347,1-39 (1938). 